\title{
PSEUDOMONAS INFECTION OF THE TEMPOROMANDIBULAR JOINT (TMJ) HEALTH AND PUBLIC HEALTH ISSUES
}

\author{
Malachovsky $\mathrm{I}^{1}$, Janickova $\mathrm{M}^{1}$, Stasko $\mathrm{J} \mathrm{JR}^{1}$, KASAJ $\mathrm{M}^{1}$, Sadlonova $\mathrm{V}^{2}$, Novakova $\mathrm{E}^{2}$, Gengelova \\ $\mathrm{P}^{1}$, Smatanová $\mathbf{M}^{1}$, Statelová D. ${ }^{1}$ \\ ${ }^{1}$ Clinic of Stomatology and Maxillofacial Surgery, Comenius University, Jessenius Faculty of Medicine \\ and University Hospital Martin, Slovakia \\ ${ }^{2}$ Institute of Microbiology, Comeniu University, Jessenius Faculty of Medicine, Martin, Slovakia
}

\begin{abstract}
The authors describe a case of a rare infectious disease of intra-articular tissues of the temporomandibular joint caused mainly by Pseudomonas aeruginosa. In scientific literature, under the heading invasive (malignant) external otitis, we can find cases of an infectious disease of the external acoustic meatus caused by a microbial agent of Pseudomonas aeruginosa which can subsequently penetrate into structures. However, a primary affliction of the abovementioned structures has not been described. Localisation and severity of the infection requires long-term and massive treatment with antibiotics.
\end{abstract}

Keywords: infectious disease of the temporomandibular joint, TMJ, Pseudomonas aeruginosa, antibiotic treatment

\section{INTRODUCTION}

Inflammatory diseases in the temporomandibular joint (further on only TMJ) region are quite rare but very complicated. In the scientific literature we come across the term invasive (malignant) external otitis, which was introduced into clinical practice by Chandler in 1968 (in Drew, 1993, Midwinter, 1999, Mardinger, 2003). This can develop into a life-threatening infection that starts in the external acoustic meatus and spreads into the surrounding soft or hard tissues. Sometimes it can penetrate into osseous tissues of the base of the skull and trigger an osteomyelitic process. If left untreated in its early stages, the disease can lead to death, which is why the term malignant is used. The infectious disease always starts after a previous trauma in the area of the external acoustic meatus with a damaged cartilaginous or osseous part of the meatus. The presence of a staphylococcal infection in the early stages of the disease has been proven by microbial examination. In the later course of the disease, Pseudomonas aeruginosa becomes the dominant microorganisms. In spite of the close anatomical position of the external acoustic meatus and TMJ structures, primary damage of TMJ structures has not been reported in the scientific literature.

\section{Case report}

In February 2008, a 20-year-old female patient came to the Centre for Painful Joints of the Clinic of Stomatology and Maxillofacial Surgery, University Hospital in Martin, complaining of pains on both sides of the TMJ that had lasted for one year. While taking the patient's history, we found out that the patient had had an injury four years previously. She had fallen down on her chin and suffered damage to the soft tissue of the face without evident trauma to the articular processes of the mandible. From the patient's other medical records, we dis-

\footnotetext{
Address for correspondence:

Doc. MUDr. Dagmar Statelová, CSc., Clinic of Stomatology and Maxillofacial Surgery, Comenius University, Jessenius Faculty of Medicine and University Hospital, Kollarova Str.N. 2,036 59 M A R T I N, Slovak Republic e-mail: Statelova@jfmed.uniba.sk
} 
covered that she was registered in the Chronic Allergy Centre due to a polyvalent drug allergy and allergies to grass, pollen, feathers, animal hair (fur) and eggs. She had also visited the Endocrine Outpatient Department with diffuse struma (no increase in anti-thyroid antibodies at that time), and the Diabetes Centre with insulin-dependent diabetes mellitus. She had also been treated by a gastroenterologist due to the presence of Helicobacter pylori and antral gastritis.

On examination, acoustic phenomena were found throughout the whole course of the opening cycle of the mandible on the right side. Palpation found pathological changes in the right disc area; during opening and closing movements the mandible deflected more to the right side. The range of the interincisal distance was approximately $38 \mathrm{~mm}$. On the basis of a preliminary diagnosis: right disc dislocation without reposition, the patient was sent for an arthroscopic examination of the right TMJ under total anaesthesia. The arthroscopic examination revealed damage to the internal part of the joint; widespread adhesions (that probably arose as a consequence of the trauma) were detected and the disc itself was rather deformed, in fact, almost ruptured. Lysis, lavage and debridement of the discus and the whole articular space were performed. A sample was taken for histo-pathological testing. The conclusion: discus degeneration of a post-traumatic aetiology.

On the basis of the abovementioned facts the patient was sent for nuclear magnetic resonance (NMR) examination, where the clinical finding was confirmed. The examination confirmed a massive deformation of the intra-articular disc with a diminished almost perforated inter-medial zone, and dislocated dorsal and medial disc fragments. The fissure was narrowed, the joint head disfigured with subcortical sclerosis present, but no physiological reduction had occurred. The examination provided us with a picture of advanced internal derangement of the grades IV - V secondary Wilkes with visible secondary arthrotic changes (Fig. $1 \mathrm{a}$ and $1 \mathrm{~b})$.
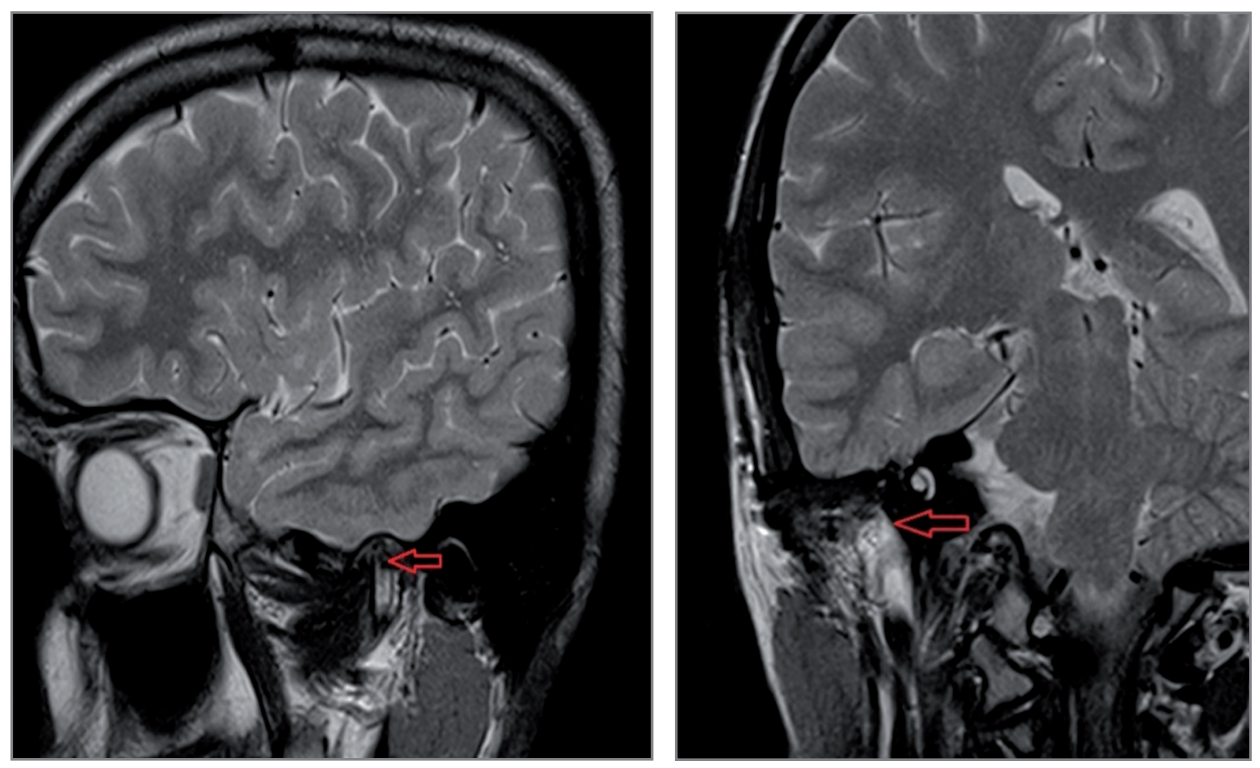

Fig. 1a, $1 b$. NMR examination of TMJ before therapy

With regard to the NMR results, it was recommended that the patient undergo arthrotomy with discectomy and high condylectomy. After careful consideration and providing her consent, the patient was operated on under total anaesthesia. The histological test confirmed the preliminary diagnosis and revealed the presence of disc fragments with chondroid metaplasia, ossification and dystrophic changes (Fig. $2 \mathrm{a}$ and $2 \mathrm{~b}$ ). 

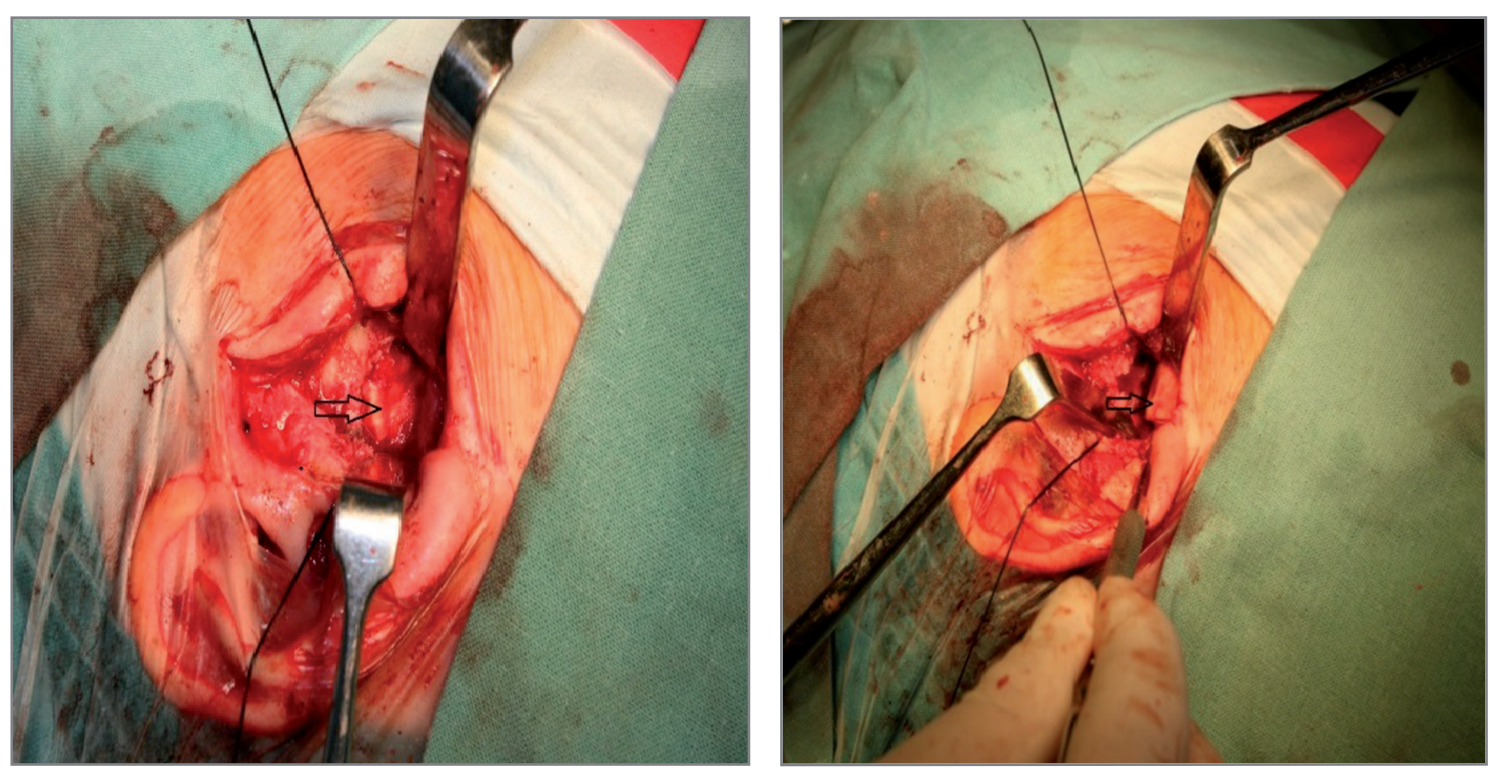

Fig. 2a, 2b. Peroperational view of demaged TMJ, state after menisectomy and high condylectomy

The wound healed as planned and the patient was discharged to outpatient care. On the ninth day after the operation the wound became inflamed and a fistula with purulent secretions developed near the auricle on the right side. Klebsiela oxytoca and Pseudomonas aeruginosa with a qualitative determination of antibiotic sensitivity. The therapy continued with cephixim (Suprax) and clindamycin (Dalacin) was added to cover suspected anaerobic complication.

After 1 month of ambulatory therapy with antibiotics there was no significant success in the treatment, thus, the patient was hospitalised and the surgeons attempted to remove the fistula from the operation wound. During the operation, under total anaesthesia, the fistula was removed and it was discovered that the inflammatory process was in the epifascial region but that the joint capsule was not afflicted. The granular tissue from the fistula area contained remnants of stitching material and had to be histo-pathologically examined. The examination revealed not only remnants of stitching material, but also an unspecific tissue around this material and noticeable evidence of an inflammatory process. Microbiological testing of a sample of this material confirmed the sporadic occurrence of Pseudomonas aeruginosa. On the basis of an antibiogram, which stated qualitative and quantitative sensitivity to antibiotics, the patient was prescribed cephalosporine antibiotics with a total daily dose of $4 \mathrm{~g}$ in combination with fungicidal preparations because the mycotic infection could not be excluded in diabetic patient and complicated course of healing process.

After 2 weeks of parenteral therapy with the abovementioned antibiotics, treatment had to be prematuraly interrupted because the patient had gastrointestinal problems. The local finding was stabilised, witht no secretions, and the patient ceased to complain of anything. However, after about another 5 months, a fistule appeared in the area of the lower pole of the previous operation scar (Fig. 3).

A CT scan revealed periostal osseous formations in the frontal part of the articular hole as well as in the area of the eminentia articularis. In the condyle process, the trabecula was thickened with several subchondral cystic lesions. The joint fissure was asymmetric and irregularly widened (Fig. 4). 


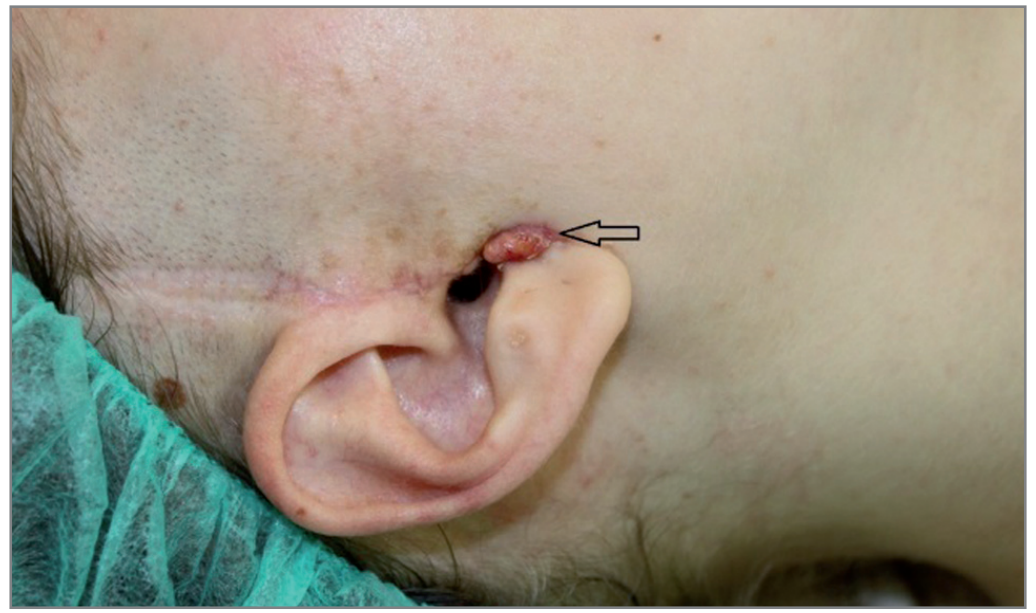

Fig. 3 Clinical picture of cutaneous fistulae

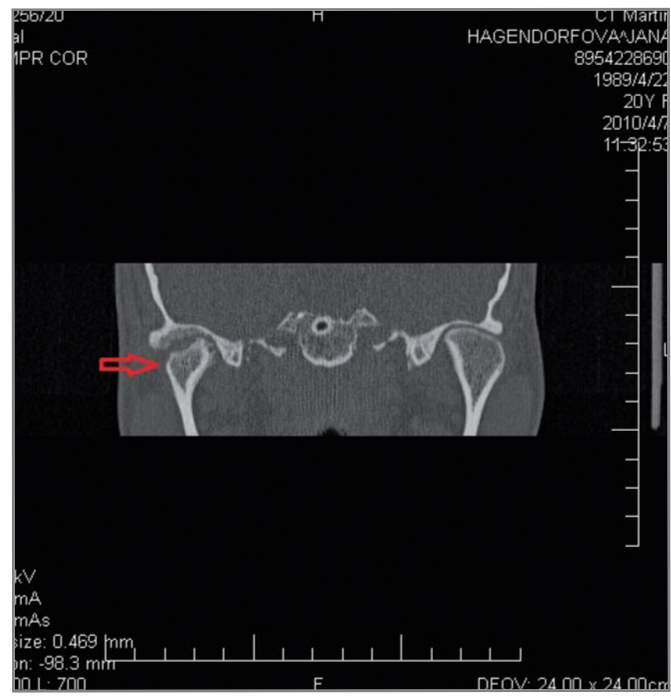

Fig. 4 TMJ CT image present periostal formations and subchondral cystic lesions

As the patient complained of severe pains and problems with jaw movements, another preoperation examination and revision of the whole TMJ area had to be performed. We again took samples for histo-pathological and microbiological tests. Inspection of the intra-articular region revealed numerous adhesions that had developed after the previous operation, but no other pathological changes. After the adhesions were removed, gentamicin foam (Garamycin) was applied to the intra-articular region. The histo-pathological tests revealed metaplasia of the chondroid tissue, regressive proliferative changes of the joint process and an unspecific inflammation of soft tissues with an abscess.

The microbiological tests repeatedly confirmed the presence of Pseudomonas aeruginosa in some places. Due to the location and persistence of the infection, we consulted a clinical microbiologist and an infectiologist, who both insisted on long-term antibiotic therapy. To allow for in vitro sensitivity and good penetration into the bone, ciprofloxacin (Ciprinol $1000 \mathrm{mg}$ per day tablet) was recommended along with a monthly check-up of biochemical parameters.

The patient is now in her sixteen month with no subjective problems and with no objective clinical signs of disease relapse (Fig. 5a and 5b). 


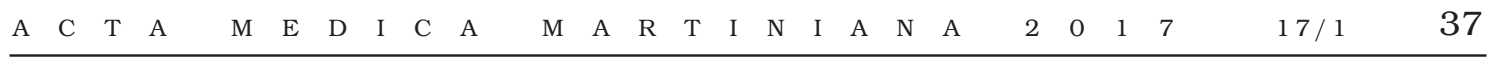

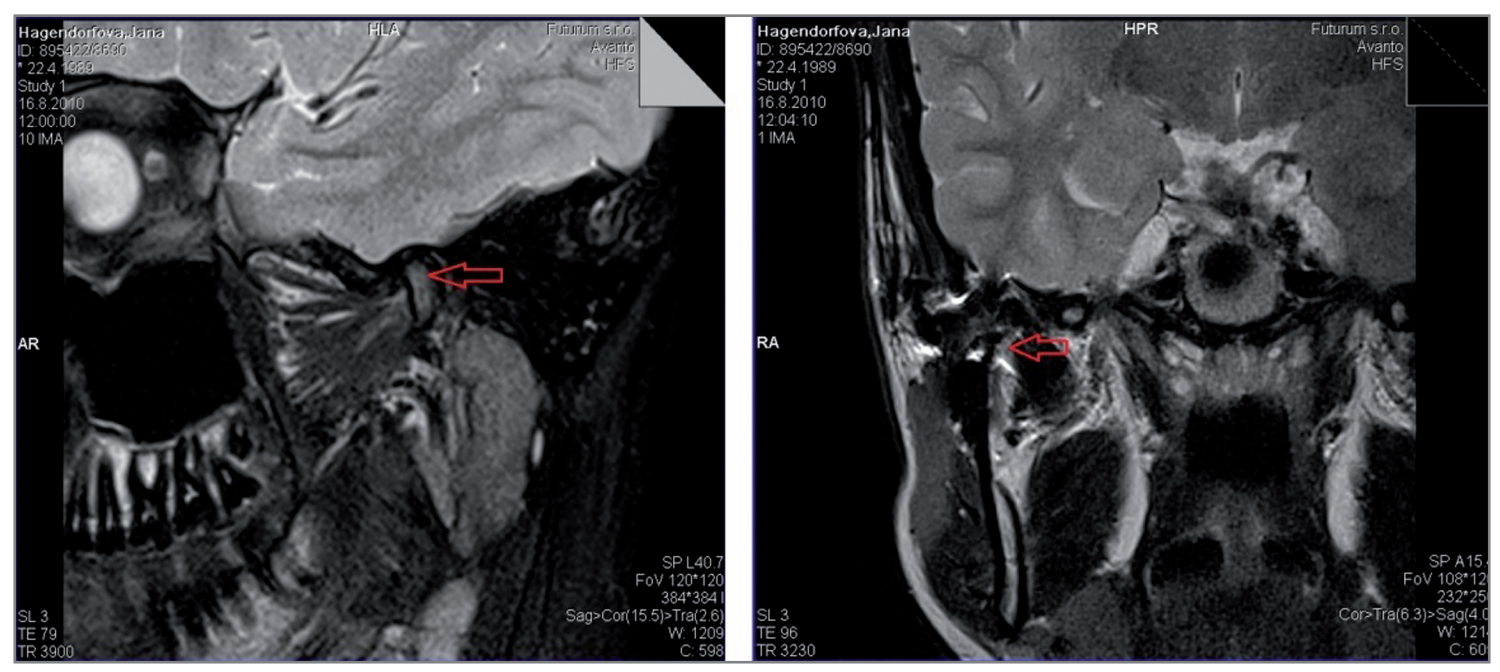

Fig. 5a, 5b. NMR examinations of TMJ 16 months after therapy

\section{DISCUSSION}

The infectious disease described as malignised invasive external otitis primarily afflicts soft tissues in the outer acoustic meatus region. However, it can spread into surrounding tissues and result in cranial neuropathy, even osteomyelitis of the base of the skull bones or TMJ structures. If treatment does not start immediately, the illness can be fatal, hence the term 'malignised' (Drew, 1993,Hollis, 2011, Lancaster, 2000,Midwinter, 1999, Mardinger, 2003, Okpala, 2006, Thompson, 2010, Vourexakis, 2010).

The infection usually starts after a traumatic damage to soft tissues. The dominant pathogen is always Pseudomonas aeruginosa; in the early stages of the disease Staphylococcus aureus can also sometimes be cultivated (Ahmad, 2006, Mehrota, 2011, Tong, 2008).

Pseudomonas produces bacterial protease enzymes and stimulates release of neutrophil proteases during infection, that are responsible for tissue destruction. In isolated cases they can lead to paralysis of cranial nerves and thrombosis of vena jugularis. In cases of a massive infection, this can spread up to cerebral tissues and cause abscesses, which can lead to death if treatment is non-existent or ineffective ( Acar, 2010, Ali, 2010, Mardinger, 2003, Rowlands, 2002, Sharma, 2011, Thomson, 2010).

On the basis of a literature review, Mardinger et al. (2003) described damage of TMJ structures in only 16 cases over a period of 50 years. In his work, he reported the occurence

of TMJ disease of up to $14 \%$.

Doroghazi et al. (1981, in Drew, 1993) described how, in a group of 21 patients with symptoms of external invasive otitis, 5 patients had symptoms of TMJ damage (Ismail, 2004).

Symptoms concerning TMJ structures can be hidden behind other pathological symptoms of the surrounding tissues, which is why each swelling in the auricular region with hypomobility of the jaw requires special attention. In spite of the relatively close proximity of the TMJ with the outer acoustic meatus, TMJ problems are very rare. The TMJ can be afflicted after repeated infections or after maltreatment (Al-Zahrani, 1993, Drew, 1993, Klein, 1988, Mercuri, 2011, McGuire, 1985).

The therapy lies in radical surgery aimed at removing necrotic parts and in targeted antibiotic treatment ( Carpenter, 2011, Drew, 1993, Mardinger, 2003, Dobbyn, 2005, Thompson, 2010). On one side there is a group of authors who prefer conservative prolonged antibiotic therapy, while on the other side there is another group of authors who prefer surgical intervention, espe- 
cially in cases with abscess deposits or an osteomyelitic process in the area of the condylar process or joint socket. Treatment should be extensive in order to preserve joint structures as much as possible ( Rowlands, 2002, Sharma, 2011).

In cases of disease relapse, Mardinger et al. (2003) successfully used hyperbaric oxygenotherapy.

Each examination of an inflamed TMJ must be accompanied by an examination of the outer acoustic meatus or a complete otologic examination (Alva, 2009, Drew, 1993, Midwinter, 1999, Mardinger, 2003, Dobbyn, 2005, Thompson, 2010).

A differential diagnosis should consider all bacterial, fungal and viral infectious processes (Eveleigh, 2009, Paul, 2005).

\section{CONCLUSION}

Temporomandibular (TMJ) infection caused by Pseudomonas aeruginosa is a rare inflammatory disease with an unpleasant course. In taking the patient's history, the doctor should place emphasis on an otologic disease examination, especially in cases with soft tissue swelling located in the area of the outer acoustic meatus in older people, in patients with diabetes mellitus, in patients after previous trauma in the TMJ area and in patients with unclear aetiology of TMJ inflammatory disease. Treatment of this inflammatory disease consists of radical surgery in combination with targeted and long-lasting antibiotic therapy. During this period of antibiotic therapy, biochemical parameters and the internal environment should be regularly monitored in each patient, especially in cases where the infectious agent Pseudomonas aeruginosa has been confirmed.

\section{REFERENCES}

1. Acar B, Guler G. et al. Isolated itching of external auditory canal: clinicopathological study with immunohistochemical determination of antimicrobial peptides. J Laryngol Otol, 2010, 125, 227-230.

2. Ahmad N, Etheridge C. et al. Prospective study of the microbiological flora of hearing aid moulds and the efficacy of current cleaning techniques. J Laryngol Otol, 2006, 121, 110-113.

3. Alva B, Chandra-Prasad K. et al. Temporal bone osteomyelitis and temporoparietal abscess secondary to malignant otitis externa. 2009, 123, 1288-1291.

4. Al-Zahrani A, Al-Moberik A. A Cauliflower ear: an unusual complication of TMJ surgery. The Saudi Dent. Journal 1993, 5, 3, 142-144.

5. Carpenter CR, Schuur JD. et al. Evidence-based diagnostics: adult septic arthritis. Acad Emerg Med 2011, 18, 781-796.

6. Dobbyn L. et al. Malignant (invasive) otitis externa involving the temporomandibular joint. J Laryngol Otol, 2005, 119, 61-63.

7. Drew SJ, et al. Invasive (malignant) external otitis progressing to osteomyelitis of the temporomandibular joint: a case report. J Oral Maxilofac Surg 1993, 51, 429-431.

8. Eveleigh MO, et al.: Prognostic scoring in necrotising otitis externa. J Laryngol Otol, 2009, 123, 10971102.

9. Haug RH, Picard U. et al. The infected prosthetic total temporomandibular joint eplacement: report of two cases. J Oral Maxillofac Surg 1989, 47, 1210-1214.

10. Hollis S, Evans K. Management of malignant (necrotising) otitis externa. J Laryngol Otol, 2011, 125, 1212-1217.

11. Ismail H, Hellier WP. et al. Use of magnetic resonance imaging as the primary imaging odality in the diagnosis and follow-up of malignant external otitis. J Laryngol Otol, 2004, 118, 576-579.

12. Klein RS. Joint infection, with consideration of underlying disease and sources of bacteremia in hematogenous infection. Clin Geriatr Med 1988, 4, 375-394.

13. Lancaster J, Alderson DJ. et al. Non-pseudomonal malignant otitis externa and jugular foramen syndrome secondary to cyclosporine induced hypertrichosis in a diabetic renal transplant patient. J Laryngol Otol, 2000, 114, 366-369. 
A $\quad$ C $\quad$ T $A$

14. Mardinger O, Rosen D. et al. Temporomandibular joint involvement in malignant external otitis. Oral Surg Oral Med Oral Pathol 2003, 96, 4, 398-403.

15. McGuire NM, Kaufman CA. Septic arthritis in the elderly. J Am Geriatr Soc 1985, 33, 170-174.

16. Mehrota P, Elbadawey MR. et al. Spectrum of radiological appearances of necrotising external otitis: a pictorial review. J Laryngol Otol, 2011, 125, 1109-1115.

17. Mercuri LG, Psutka D. Perioperative, postoperative and prophylactic total temporomandibular joint replacement surgery: a survey and preliminary guidelines. J Oral Maxillofac Surg 2011, 69, 21062111.

18. Okpala NCE, Siraj $\mathrm{QH}$. et al. Radiological and radionuclide investigation of malignant otitis externa. J Laryngol Otol, 2006, 119, 71-75.

19. Paul CA, Kumar A. et al. Pseudomonas cervical osteomyelitis with retropharyngeal abscess: an unusual complication of otitis media. J Laryngol Otol, 2006, 119, 816-818.

20. Rowlands RG, Lekakis GK. et al. Masked pseudomonal skull base osteomyelitis presenting with a bilateral Xth cranial nerve palsy. J Laryngol Otol, 2002, 116. 556-558.

21. Sharma A, Deshmukh S. et al. Wegenerś granulomatosis mimicking skull base osteomyelitis. J Laryngol Otol, 2011, 126, 203-206.

22. Thompson ACM. et al. Necrotising otitis externa: an unusual cause of cranial nerve palsy in a diabetic haemodialysis patient. J R Coll Physicians Edinb 2010, 40, 26-28.

23. Tong D, Theis JC. Antibiotic prophylaxis and invasive dental treatment in prosthetic joint patients. N Z Med J 2008, 22, 45-52.

24. Vourexakis Z, Kos MI. et al. Atypical presentations of malignant otitis externa. J Laryngol Otol, 2010, 124, 1205-1208.

Received: March, 23, 2017

Accepted: April, 6, 2017 